\title{
The medical unit round: engaging trainees in curriculum- relevant teaching
}

\author{
Author: Aarij S Siddiqui
}

\section{Aims}

To provide weekly curriculum-relevant teaching for medical juniors as per Joint Royal Colleges of Physicians Training Board (JRCPTB) quality criteria for core medical trainees.

\section{Methods}

Previous attempts at establishing sustainable weekly medical teaching at our hospital had failed secondary to poor attendance from both trainees and consultants. At the beginning of the year, the Royal College of Physicians college tutors, associate college tutors and chief registrar agreed that re-establishing high quality weekly teaching was a high priority in order to improve trainee experience and satisfy JRCPTB requirements.

We investigated reasons as to why attendance at previous medical teaching sessions had been poor. After speaking with junior doctors and consultants we identified that ward commitments, lack of support from consultants, poor-quality teaching sessions and no lunch provided were key reasons for poor attendance.

In November 2017 we set up the 'medical unit round'; a series of weekly medical teaching sessions delivered by consultants and registrars for junior doctors in training. Lunch was provided and the topics for each session were publicised via emails and WhatsApp messages on the day. Topics for presentation were selected from the core medical training and general internal medicine curricula in addition to topics that trainees had suggested would be beneficial. Emphasis was placed on providing trainees with relevant focused teaching that would be useful in an acute or on-call scenario.

Junior doctors were encouraged to make a reflective ePortfolio entry during each teaching session, in addition to uploading the provided three-monthly attendance certificates into their online library. The presenters were also provided with certificates documenting their contribution to the teaching programme.

\section{Results}

Since November 2017 we have held 15 medical unit rounds, with an average attendance of 18 people (11 to 29). The feedback has been positive and junior doctors are keen to attend the sessions.

Author: Royal College of Physicians chief registrar, Cardiff and Vale University Health Board, Wales, UK
The sessions have also been used as an informal junior doctors' forum, allowing the chief registrar to engage with trainees and allow any concerns or issues to be discussed. Future work includes making these sessions 'bleep-free'.

\section{Conclusions}

By providing teaching on topics relevant to trainees, free lunch and certificates for the ePortfolio, we have been able to sustain wellattended weekly medical teaching sessions. These sessions have provided an added advantage for the chief registrar, in terms of engagement with trainees.

\section{Conflict of interest statement}

None declared. 